\title{
Observations on the rare association between portal and pulmonary hypertension
}

\author{
GOLAM KIBRIA, PAUL SMITH, DONALD HEATH, AND SHANTI SAGAR
}

\begin{abstract}
From the Departments of Pathology and Surgery, University of Liverpool, Royal Liverpool Hospital, Liverpool
\end{abstract}

ABSTRACT Pulmonary arterial hypertension with severe pulmonary vascular disease is a rare association of portal hypertension in man, be it the result of cirrhosis of the liver or obstruction of the portal vein. We induced portal hypertension in 23 rats by partially ligating the portal vein or by totally occluding it in two stages. The rats were killed between one and 15 months after operation. A collateral circulation of varicose, anastomotic vessels was established, and in six animals wellmarked oesophageal varices developed. Despite this evidence of sustained portal hypertension, hypertrophy did not develop in the right ventricle or in the media of the pulmonary trunk or muscular pulmonary arteries in any of the animals. This suggests that mechanical obstruction of the portal vein per se is not responsible for the development of pulmonary hypertension. Other factors, perhaps of a humoral nature, appear to be required to induce this rare association of portal hypertension but we have been unable to identify these. In particular, blood levels of oestrogen were not raised after ligation of the portal vein.

Very rarely cases of portal hypertension after cirrhosis of the liver or thrombosis of the portal vein in man become complicated by severe pulmonary arterial hypertension. The association was first reported by Mantz and Craige, ${ }^{1}$ and a series of six such cases was described by Naeye. ${ }^{2}$ The elevation of pulmonary arterial blood pressure associated with portal hypertension is accompanied by the form of hypertensive pulmonary vascular disease designated "plexogenic pulmonary arteriopathy," ${ }^{3}$ characterised by plexiform lesions whose nature and possible association with fibrinoid necrosis and pulmonary vasoconstriction we have described ${ }^{4}$ recently. Workers in our group have previously attempted to induce in rats pulmonary hypertension and associated pulmonary vascular disease by first inducing cirrhosis of the liver and portal hypertension. ${ }^{5}$ They successfully induced cirrhosis of the liver and portapulmonary venous anastomoses but not pulmonary arterial hypertension. From this it was concluded that an elevation of pulmonary arterial blood pressure is not produced by hepatic cirrhosis and portal hypertension per se but requires some additional factor which

Address for reprint requests: Professor D Heath, Department of Pathology, Duncan Building, Royal Liverpool Hospital, Liverpool L7 8XW. appears to develop only rarely in cases of cirrhosis of the liver. To support or refute this hypothesis we thought it would be of interest to see if experimental ligation of the portal vein always leads to pulmonary hypertension or whether, as in the case of hepatic cirrhosis, production of portal hypertension as a result of the local hepatic lesion is insufficient to induce pulmonary vascular disease and demands the addition of an as yet undetermined factor.

\section{Methods}

Two separate experimental techniques were employed to produce portal hypertension in rats. The first produced partial blockage of the portal vein whereas the second created a gradual but total occlusion of the vessel.

In the first experiment 33 adult Wistar Albino rats of both sexes were used. They were anaesthetised with ether and a midline incision made over the anterior third of the abdomen. The portal vein was isolated from its tethering fascia and a loop of grade $4 / 0$ braided silk suture thread passed under the vessel close to the liver. A steel wire measuring $1.1 \mathrm{~mm}$ in diameter was then placed alongside the vessel and a suture tied firmly but not too tightly around both the 
vein and the wire. The wire was then withdrawn allowing the vein to expand slightly into the loose ligature. This technique for inducing a stenosis of the portal vein was first described by Whitaker ${ }^{6}$ and later modified by Cheever and Warren. ${ }^{7}$ The diameter of the wire was chosen at $1.1 \mathrm{~mm}$ since this produces the maximum degree of stenosis compatible with survival in the adult rat. ${ }^{8}$ After ligation the abdomen was closed with a continuous suture and the wound sealed with plastic skin ("Nobecutane").

In the second experiment partial ligation of the portal vein was carried out as described above. In addition to the first ligature a second loop of suture thread was passed round the vein and loosely knotted with a surgeon's knot. The ends of the thread were brought to the surface of the abdomen and passed through the body wall laterally. They were inserted between the body wall and the skin before the abdominal incision was sutured. The animals were then allowed to recover and kept for 10 days to permit a collateral circulation to develop. A second operation was then performed in which the abdomen was opened, the two ends of suture thread located and pulled firmly to tighten the loose knot around the portal vein. A two-stagc total ligation of the portal vein was performed in this manner on 13 rats. A control group of 14 rats were subjected to a sham operation. In this the abdomen was opened, the portal vein separated from its fascia and manipulated with forceps. The abdomen was then closed and sutured in the same manner as the test rats.

In the first experiment 10 rats died within 24 hours of operation and a further four died within a week. After this period recovery was completc and the remaining 19 animals could be used to study the long-term effects of the ligation. They were killed at intervals ranging from one month to one year three months with 11 animals surviving for one year or longer. After the double ligation procedure only four rats survived the second operation and these were killed after 11 months.

All rats surviving the operation and the control rats were anaesthetised with ether and a sample of blood taken from the inferior vena cava. This was centrifuged and the plasma stored in a deep freeze for subsequent assay of oestrogen. The thorax was opened and the thoracic organs removed intact. The lungs were distended with formalin and the thoracic organs fixed in a similar solution. Tissue was also taken from the small intestine, spleen, liver, adrenal, and oesophagus. The portal vein was also resected.
After fixation the heart was removed from등 other thoracic organs and its ventricles dissected $\overline{\bar{N}}$ free from one another. These were weighed and $\vec{\Phi}$ the weight of the left ventricle expressed as $a$ ratio of that of the right ventricle in order to detect the presence of right ventricular hyper- $\vec{O}$ trophy. Histological sections of the lungs were $\vec{A}$ stained by an elastic Van Gieson method and ${ }_{\sigma}^{\omega}$ the medial thickness of their muscular pulmonary $\vec{f}$ arteries measured by the method of Kay and ${ }_{\omega}^{x}$ Heath. ${ }^{9}$ Transverse sections of pulmonary trunk and aorta were similarly stained and their $\vec{\sim}$ medial thickness measured. The thickness of the pulmonary trunk was expressed as a ratio of 0 that of the aorta.

The blood plasma was assayed for the level of $\rightarrow$ oestradiol using a radioimmunological method. The oestradiol in the plasma was labelled with $\Phi_{\mathscr{\Phi}}$ ${ }^{12.5}$ I. Antiserum to oestradiol was raised in the $\frac{3}{6}$ rabbit and added to the labelled plasma. The bound fraction was precipitated with polyethylene $\vec{\oplus}$ glycol and its level of radioactivity determined. $\%$

\section{Results}

\section{MACROSCOPIC FINDINGS}

In those rats which died a few hours after $\frac{\mathscr{Q}}{\mathbb{Q}}$ operation the portal vein and its tributaries $\stackrel{\circ}{\vec{a}}$ proximal to the ligature were greatly dilated. $\overrightarrow{\overrightarrow{0}}$ The intestines were grossly congested and oedematous. The liver showed no abnormality apart from being slightly paler than normal. Death was attributed to the acute effects of portal vein ligation. ${ }^{1011}$ In rats surviving longer $\frac{0}{9}$ than 24 hours but less than one week, death was: caused in all but one case by rupture of collateral 3 vessels in the omentum causing massive haemoperitoneum. In these animals the intestines윽 and spleen were congested and the liver was pale with white patches of necrosis. Only onc? animal died from sepsis of the operation site: $\frac{D}{0}$ The thoracic organs were normal. In animals which died after the double ligation procedure. $\%$ death was also caused by rupture of collateralin vessels in the omentum. In these animals con-N gestion of the intestines and spleen was less severe.

In rats which survived longer than a week? the necropsy findings were similar irrespective of $\stackrel{\mathbb{Q}}{\mathcal{D}}$ the period of survival or the type of operation. Examination of the portal vein was hampered by the presence of dense adhesions between it and lobes of the liver or loops of the bowel. The $\frac{\rho}{\mathrm{Q}}$ region of the ligature was surrounded by a dense $\varrho$ nodule of fibrous tissue. The portal vein proximate to the obstruction was not distended, indeed ito 
was often narrower than in the control rats. Most striking were numerous distended, often varicose collateral vessels in the omentum. These could be traced either directly to the liver or to the portal vein distal to the ligature thus bypassing the obstruction. Collateral vessels in the pleura could not be found but oesophageal varices were seen in six rats killed after 11 months, two after total ligation and four after partial ligation. All other organs were normal except for the spleen which was enlarged (table 1).

\section{HISTOLOGICAL FINDINGS}

In rats which died prematurely the main histological feature was gross congestion of the intestines and spleen. In the intestine there was often associated haemorrhage within the submucosa. In the liver the hepatocytes were vacuolated with small foci of necrosis or occasional, larger infarcts. Vacuolation of hepatocytes was also seen in rats with prolonged survival up to approximately two months, after which time the liver was identical to that in control rats. The histology of all other organs in the surviving rats from both groups was normal. Histological examination of the portal

Table 1 Mean values of measurements on the heart, great vessels, pulmonary arteries, and spleen in test and control rats

\begin{tabular}{|c|c|c|c|c|}
\hline Measurement & Control & Test & $t$ & Significance \\
\hline $\begin{array}{l}\text { Final body weight } \\
\text { (g) }\end{array}$ & $\begin{array}{c}533 \\
(175)\end{array}$ & $\begin{array}{c}493 \\
(147)\end{array}$ & 0.522 & NS \\
\hline $\mathbf{L V}+\mathbf{S}(\mathrm{g})$ & $\begin{array}{c}0.931 \\
(0 \cdot 228)\end{array}$ & $\begin{array}{c}0.912 \\
(0.219)\end{array}$ & $0 \cdot 116$ & NS \\
\hline$R V(g)$ & $\begin{array}{c}0 \cdot 236 \\
(0 \cdot 074)\end{array}$ & $\begin{array}{c}0 \cdot 234 \\
(0 \cdot 057)\end{array}$ & $0 \cdot 811$ & NS \\
\hline$\frac{\mathrm{LV}+\mathrm{S}}{\mathrm{RV}}$ & $\begin{array}{c}4 \cdot 05 \\
(0 \cdot 52)\end{array}$ & $\begin{array}{c}3.93 \\
(0 \cdot 46)\end{array}$ & 0.729 & NS \\
\hline $\mathrm{PT}(\mu \mathrm{m})$ & $\begin{array}{c}64 \\
(14)\end{array}$ & $\begin{array}{c}66 \\
(17)\end{array}$ & 0.500 & NS \\
\hline$A(\mu m)$ & $\begin{array}{l}152 \\
(37)\end{array}$ & $\begin{array}{l}157 \\
(30)\end{array}$ & $0 \cdot 594$ & NS \\
\hline$\frac{P T}{A}$ & $\begin{array}{c}0.42 \\
(0.05)\end{array}$ & $\begin{array}{c}0.42 \\
(0 \cdot 10)\end{array}$ & 0.000 & NS \\
\hline MT $\%$ & $\begin{array}{c}3 \cdot 6 \\
(0 \cdot 5)\end{array}$ & $\begin{array}{c}3 \cdot 4 \\
(0 \cdot 6)\end{array}$ & $1 \cdot 039$ & NS \\
\hline Splenic weight (g) & $\begin{array}{c}0.744 \\
(0 \cdot 275)\end{array}$ & $\begin{array}{c}1.004 \\
(0 \cdot 292)\end{array}$ & $2 \cdot 665$ & $\mathrm{p}<0.025$ \\
\hline $\begin{array}{l}\text { Splenic weight } \times \\
10^{3} \div \text { body weight }\end{array}$ & $\begin{array}{l}1 \cdot 4 \\
t(0 \cdot 2)\end{array}$ & $\begin{array}{l}2 \cdot 1 \\
(0 \cdot 4)\end{array}$ & $6 \cdot 462$ & $\mathrm{p}<0.001$ \\
\hline
\end{tabular}

$\mathbf{L V}+\mathbf{S}=$ weight of the left ventricle plus the interventricular septum. $\mathbf{R V}=\quad$ weight of the right ventricle;

PT $=$ medial thickness of the pulmonary trunk;

$A=\quad$ medial thickness of the ascending aorta;

$\mathrm{MT} \%=$ mean medial thickness of muscular pulmonary arteries expressed as a percentage of their external diameter;

$t=\quad$ Students $^{\prime} t$ test for comparison of means:

Figures in parentheses represent one standard deviation from the mean. vein confirmed that there was a stenosis in the first group, or total obstruction to the vessel in the second. The fibrous tissue surrounding the ligature often contained numerous vein-like sinuses running roughly parallel to the portal vein.

\section{QUANTITATIVE DATA}

The quantitative findings from the two groups of test rats and the control rats are summarised in table 1 . Since there were no differences in any of the parameters between the two groups of test animals, the measurements from both have been combined in the tables. There was no evidencc of pulmonary hypertension as assessed by measurements of right ventricular weight, medial thickness of the muscular pulmonary arteries, or the ratio of the thickness of the pulmonary trunk to that of the aorta.

The levels of oestrogen in the blood were not raised after partial ligation of the portal vein. The oestradiol concentrations in male rats are shown in table 2 . There was considerable variation in these concentrations between individual animals in both test and control groups. The mean value in test rats was almost half that in the control rats but this difference showed no statistical significance.

\section{Discussion}

There is no doubt that portal hypertension was induced in the test rats since the portal vein become engorged and an extensive collateral circulation was stimulated. It seems to us that venous collaterals play little or no part in these rare cases of pulmonary arterial hypertension complicating portal hypertension. In previous experiments from this laboratory in which cirrhosis of the liver was induced by the inhalation of carbon tetrachloride there was an extensive

Table 2 Blood oestradiol concentration in male rats after partial ligation of the portal vein and in control rats

\begin{tabular}{lcc}
\hline Test rats $(p$ mole $l-1)$ & Control rats $(p$ mole $l-1)$ \\
\hline & $<36$ & 72 \\
& $<36$ & 198 \\
& $<36$ & 40 \\
97 & 432 \\
& 158 & 54 \\
97 & 274 \\
& 83 & 54 \\
& $\cdots$ & 68 \\
Mean & 78 & 149 \\
SD & \pm 46 & \pm 142 \\
\hline
\end{tabular}


mediastinal collateral system around the oesophagus with the formation of numerous portapulmonary venous anastomoses but there was no right ventricular hypertrophy or pulmonary vascular disease. ${ }^{5}$ While such porta-pulmonary venous anastomoses entering large pulmonary veins could readily account for the unsaturation of systemic arterial blood and cyanosis which sometimes occurs in cirrhosis of the liver, it is hard to see how an increased flow of blood entering the pulmonary circulation directly into the pulmonary veins could lead to plexogenic pulmonary arteriopathy, a form of vascular disease which in fact does not develop with raised pulmonary venous pressure. In the present experiments oesophageal varices developed in six of the animals. In other rats the collateral vessels formed a direct anastomosis from the omentum either to the liver or to the portal vein distal to the ligature. Under these circumstances it is likely that, far from inducing pulmonary hypertension, such venous anastomoses may even have provided an adequate bypass to the portal vein thereby relieving the portal hypertension.

An alternative hypothesis to this mechanical explanation for the onset of severe pulmonary hypertension in rare cases of cirrhosis or portal vein obstruction is that a humoral mechanism is responsible. It is well known that certain substances which are toxic to the liver will also produce pulmonary arterial hypertension in experimental animals. Examples are pyrrolizidine alkaloids such as monocrotaline, ${ }^{9}$ fulvine, ${ }^{12}$ and senecionine. ${ }^{13}$ In cirrhosis or portal vein obstruction it is possible that dietary metabolites, which are normally detoxified by the liver, bypass the liver, and find their way to the lungs. For example, the level of oestrogen in the blood is sometimes raised in cirrhosis. In our test animals we found that the ligation procedure did not lead to an elevation of oestrogen levels in the blood.

Occasionally in man, obstruction of the portal vein leads to nodules of adenomatous hyperplasia in the liver. ${ }^{14}{ }^{15}$ In the latter case the obstruction was associated with fatal plexogenic pulmonary arteriopathy. It is conceivable that such nodules of adenomatous hyperplasia secrete an abnormal metabolite that might have a vasoconstrictive influence on the pulmonary arteries. Although these nodules have been induced experimentally in rats by creating a porta-caval anastomosis, ${ }^{16}$ simple obstruction of the portal vein in our study did not lead to their appearance.

Hence we are faced with the fact that four rats surviving for 11 months after complete $\frac{0}{5}$ ligation of the portal vein and 11 rats sur- $\frac{\bar{D}}{\frac{1}{3}}$ viving for a year or more after partial liga- $\bar{\phi}$ tion of the vessel did not develop pulmonary $\frac{\circ}{0}$ hypertension. On the other hand we have $s$ recently reported a case where obstruction and $\overrightarrow{0}$ recanalisation of the portal vein led to fatal $\vec{\overrightarrow{ }}$ pulmonary hypertension in a young man of $19 \vec{\omega}$ years. ${ }^{15}$ One thing that is apparent from clinical $\vec{P}$ studies is that cirrhosis of the liver is associated $x$ only very rarely with pulmonary hypertension. ${ }^{17}$ o In 12 years of practice at the Queen Elizabeth $\vec{N}$ Hospital, Birmingham, one of us (DH) saw only 0 one such case and in the next 11 years at Liver. 0 pool only the single case occurring in portal vein 은 obstruction referred to above. During these $23-$ years many cases of cirrhosis of the liver were $\mathbb{\mathbb { D }}$ seen at necropsy. This may explain why we have $\overparen{\Phi}$ failed to induce pulmonary hypertension in only $\bar{Z}$ 23 rats; perhaps a very large number of test $\stackrel{\mathbb{C}}{\stackrel{-}{ }}$ animals may be required to induce it. It would $\overrightarrow{0}$ appear that in cirrhosis of the liver or portal vein $\$$ thrombosis, some added factor is needed to induce intense pulmonary vasoconstriction which in turn leads to plexogenic pulmonary arteriopathy, that form of pulmonary vascular disease which occurs in association with large congenital $\frac{\otimes}{\varnothing}$

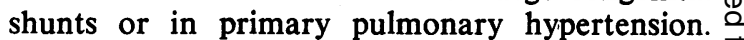
We have been unable to detect that factor from $\overrightarrow{\overline{0}}$ our experiments and its nature remains obscure to us.

We should like to thank Dr JC Davis, Director of the Sub Department of Endocrine Pathology, University of Liverpool, for performing the oestradiol assays. Dr Golam Kibria was in $\frac{0}{3}$ receipt of a Commonwealth Fellowship grant from the British Council while this work was undertaken.

\section{References}

1 Mantz FA, Craige E. Portal axis thrombosis with spontaneous portacaval shunt and resultant cor 5 pulmonale. Arch Pathol 1951; 52:91-7.

2 Naeye RL. "Primary" pulmonary hypertension N with coexisting portal hypertension. A retrospec- $\sigma$ tive study of six cases. Circulation 1960; 22: 376-84.

3 Hatano S, Strasser J, eds. Primary pulmonary $\mathbb{D}$ hypertension. Geneva: World Health Organisa- ? tion, 1975.

4 Smith P, Heath D. Electron microscopy of the plexiform lesion. Thorax 1979; 34:177-86.

5 Khaliq SU, Kay JM, Heath D. Porta-pulmonary venous anastomoses in experimental cirrhosis of $\bar{\sigma}$ the liver in rats. $J$ Pathol 1972; 107:167-74.

6 Whitaker WL. Portal vein ligation and the Eck $\delta$ 
fistula in the rat. Proc Soc Exp Biol Med 1946; 61:420-3.

7 Cheever AW, Warren KS. Portal vein ligation in mice: portal hypertension collateral circulation, and blood flow. J Appl Physiol 1963; 18:405-7.

8 Myking AO, Halvorsen JF. A method for graded portal vein stenosis in rats: survival related to degree of stenosis. Eur Surg Res 1973; 5:454-7.

9 Kay JM, Heath D. Crotalaria spectabilis, the pulmonary hypertension plant. Springfield, Illinois: Charles C Thomas, 1969.

10 Boyce FF, Lampert R, McFetridge E. Occlusion of the portal vein. $J$ Lab Clin Med 1935; 20: 935-43.

11 Johnstone FRC. Acute ligation of the portal vein. Surgery 1957; 41:958-71.

12 Kay JM, Heath D, Smith P, Bras G, Summerell
J. Fulvine and the pulmonary circulation. Thorax 1971; 26:249-61.

13 Burns $J$. The heart and pulmonary arteries in rats fed on Senecio jacobaea. J Pathol 1972; 106: $187-94$.

14 Klemperer P. Cavernomatous transformation of the portal vein. Arch Pathol 1928; 6:353-77.

15 Saunders JB, Constable TJ, Heath D, Smith P, Paton A. Pulmonary hypertension complicating portal vein thrombosis. Thorax 1979; 34:281-3.

16 Weinbren K, Washington SLA. Hyperplastic nodules after portacaval anastomosis in rats. Nature (Lond) 1976; 264:440-2.

17 Lebrec D, Capron J-P, Dhumeaux D, Benhamou J-P. Pulmonary hypertension complicating portal hypertension. Am Rev Respir Dis 1979; 120: 849-56. 